\title{
REVIEW
}

\section{Outcome of endoscopic vs microsurgical transsphenoidal resection for Cushing's disease}

\author{
Nidan Qiao ${ }^{1,2}$ \\ ${ }^{1}$ Department of Neurosurgery, Huashan Hospital, Fudan University, Shanghai, China \\ ${ }^{2}$ Harvard Medical School, Boston, Massachusetts, USA \\ Correspondence should be addressed to N Qiao: norikaisa@gmail.com or nidan_qiao@hms.harvard.edu
}

\begin{abstract}
Introduction: It is unclear whether the proportions of remission and the recurrence rates differ between endoscopic transsphenoidal surgery (TS) and microscopic TS in Cushing's disease (CD); thus, we conducted a systematic review and meta-analysis to evaluate studies of endoscopic TS and microscopic TS.

Methods: We conducted a comprehensive search of PubMed to identify relevant studies. Remission and recurrence were used as outcome measures following surgical treatment of $\mathrm{CD}$.

Results: A total of 24 cohort studies involving 1670 adult patients were included in the comparison. Among these studies, 702 patients across 9 studies underwent endoscopic TS, and 968 patients across 15 studies underwent microscopic TS. Similar baseline characteristics were observed in both groups. There was no significant difference in remission between the two groups: $79.7 \%$ (95\% Cl: $73.1-85.0 \%)$ in the endoscopic group and $76.9 \%(95 \% \mathrm{Cl}: 71.3-81.6 \%)$ in the microscopic group $(P=0.485)$. It appears that patients who underwent endoscopic surgery experience recurrence less often than patients who underwent microscopic surgery, with recurrence proportions of $11.0 \%$ and $15.9 \%$, respectively $(P=0.134)$. However, if follow-up time is taken into account, both groups had a recurrence rate of approximately $4 \%$ per person per year $(95 \% \mathrm{Cl}$ : $3.1-5.4 \%$ and $3.6-5.1 \%, P=0.651)$.

Conclusions: We found that remission proportion and recurrence rate were the same in patients who underwent endoscopic TS as in patients who underwent microscopic TS. The definition of diagnosis, remission and recurrence should always be considered in the studies assessing therapeutic efficacy in CD.
\end{abstract}

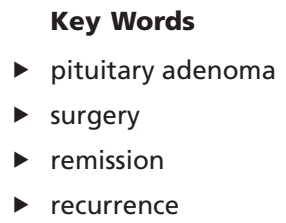

\section{Introduction}

Cushing's disease (CD) is a subtype of pituitary adenoma with hypercortisolism and presents a particular challenge to neurosurgeons. Transsphenoidal surgery (TS) has long been the standard of care for patients with $\mathrm{CD}(1,2,3,4)$. However, even under the most favorable circumstances, previous reports have found recurrence proportions of up to $10-20 \%$ after the first TS $(5,6,7)$. Recurrent or residual $\mathrm{CD}$ is associated with a threefold to fivefold increase in mortality rate $(8,9,10)$.

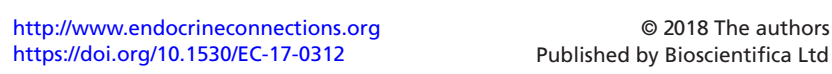

In recent decades, the application of endoscopes in the approach for treating pituitary adenomas has gained considerable popularity $(11,55,56)$. The endoscopic technique provides a panoramic surgical view with increased illumination of the anatomic structures and allows for a close-up visual examination of the suspected tumor. Different optical angles can be used to make it possible to reach the suprasellar region as well as lateral extensions $(12,13)$. Owing to these advantages, increasing 
numbers of neurosurgeons have started to adopt the endoscopic technique in recent years. Compared with microscopic surgery, endoscopic transsphenoidal tumor resection seems to lead to improved patient outcomes, especially in those patients with cavernous sinus invasion $(14,15,16,17,18,19,20)$. In patients with CD, though a few studies do indicate a lower recurrence rate $(15,21,22)$, it is still unclear whether the endoscopic technique has any advantages. Indeed, follow-up time in these studies was relatively short. It is unclear whether the proportions of remission and recurrence rates differ between endoscopic TS and microscopic TS.

The best way to compare clinical outcomes between endoscopic TS and microscopic TS is to execute a clinical trial (23), which is neither feasible nor practical due to limited sample size, variation in surgeons' experience, institutional differences and ethical considerations. Thus, to gain more insight into the potential advantages of endoscopic TS for patients with CD, especially with respect to endocrine outcomes, we conducted a systematic review and meta-analysis to evaluate studies of endoscopic TS and microscopic TS.

\section{Method}

\section{Study search strategy}

We conducted a comprehensive search ('Pituitary ACTH Hypersecretion/surgery' (Mesh) or 'CD surgery') using PubMed to identify relevant studies without limitation on language. Reference lists from studies and systematic reviews identified electronically were manually searched to identify additional eligible studies. When more than one publication shared the same patient population, we included only the most recent report in the meta-analysis.

\section{Inclusion and exclusion criteria}

We identified eligible articles based on the following inclusion criteria: (1) publication date (later than 2005); (2) study design (cohort studies); (3) target adult population (microscopic TS or endoscopic TS of CD) and (4) sufficient published data to allow for the estimation of a rate with a 95\% confidence interval (CI).

To compare the differences between patients with endoscopic surgery and patients with microscopic surgery, several exclusion criteria were employed as follows: (1) studies without endocrinology outcome or follow-up

$$
\text { http://www.endocrineconnections.org }
$$

data; (2) studies with a specific focus on a particular kind of tumor (e.g., macroadenomas or MRI-negative tumors); (3) studies that included children and/or teenagers; (4) studies with fewer than $20 \mathrm{CD}$ patients and (5) studies that included both procedures or did not mention which procedure was used.

Studies with patients treated prior to 1990 were considered separately because the follow-up period was longer in these studies. We included these studies in this meta-analysis to examine the effect of long-term follow-up on recurrence.

\section{Data extraction}

The decision about whether a study should be included was made by the author $(\mathrm{N} \mathrm{Q})$. The results were reviewed by two senior physicians (M S and X S). The data extracted included the first author's name and publication date, as well as patient age, gender composition, MRI feature, endocrine remission, follow-up time and recurrence.

The diagnosis of CD was established by Cushingoid symptoms; endogenous hypercortisolism; dynamic test, inferior petrosal sinus sampling and pituitary MRI in most studies. Positron emission tomography with 18-fluorodeoxyglucose to localize a hypermetabolic focus within the sella was used in one study (22). The term 'remission' is defined by hypocortisolism with low serum cortisol $(<5 \mu \mathrm{g} / \mathrm{dL})$ and/or low urinary free cortisol ( $<20 \mathrm{mg}$ per $24 \mathrm{~h})$, and/or low cortisol $(<1.8 \mathrm{mg} / \mathrm{dL})$ level after $1 \mathrm{mg}$ dexamethasone. Most of the studies also defined eucortisolism as remission $(15,17,18,25,28$, $29,30,31,32,33,34,36,37)$. Several studies also include the need for corticosteroid replacement and significant changes in clinical features as remission criteria $(14,25$, $30,34,35)$. The proportion of remission was calculated by dividing the number of patients with remission following surgery by the total number of patients. Half of the publications defined 'recurrence' as elevated cortisol serum level and/or elevated midnight salivary cortisol levels and/or elevated 24-h UFC levels associated with clinical symptoms of CD $(16,20,26,28,29,30$, $33,38)$. The proportion of recurrence following surgery was calculated by dividing the number of patients with recurrence by the number of patients with remission. We also estimated the recurrence rate, which was the number of patients with recurrence following surgery divided by the follow-up time (in patient-years) for patients with remission. 


\section{Statistical methods}

Demographic characteristics (age, gender, tumor volume and cavernous sinus invasion) and outcome (remission proportion, recurrence proportion and recurrence rate) between endoscopic and microsurgical approaches were compared using 'metaprop' function in R. Whether randomeffects or fixed-effects should be used was decided by the $I^{2}$ tests. 'Forest' function in $\mathrm{R}$ was used for the forest plot with subgroup analysis. The presence of heterogeneity across trials was evaluated, and a $P$ value $\leq 0.05$ was considered to be significant. Meta-regressions were performed with potential modifiers. The sensitivity analysis was also performed by removing a single study to determine the influence of that individual data set on the pooled proportions or rates. Funnel plots were also constructed to estimate the publication bias of the literature. All the statistical analyses were performed with R Studio, version 1.0.143.

\section{Results}

\section{Study characteristics}

A total of 1104 citations were identified by our search strategy. After a detailed evaluation of these articles, 80 studies remained for assessment. After applying the selection criteria, 36 cohort studies involving 4326 patients were identified and included in the meta-analysis $(14,15,16,17,18,19,20,21,22,24,25,26,27,28,29$, $30,31,32,33,34,35,36,37,38,39,40,41,42,43,44,45$, $46,47,48,49,50)$. In these 36 studies, 2656 patients were included in 12 studies $(39,40,41,42,43,44,45,46,47$, $48,49,50)$ with treatments prior to 1990 and long-term follow-up. Among the remaining 24 studies, 702 patients across nine studies underwent endoscopic TS $(14,15,16$, $17,18,19,20,21,22)$, and 968 patients across 15 studies underwent microscopic TS $(24,25,26,27,28,29,30,31$, $32,33,34,35,36,37,38)$. Baseline patient characteristics are summarized in Table 1.

\section{Demographic characteristics}

No study directly compared endoscopic and microsurgical approaches. Similar baseline characteristics were observed in both groups. The average patient age in the endoscopic group and the microscopic group was 41.3 years and 41.4 years, respectively $(P=0.981)$. Females accounted for $79.5 \%$ (95\% CI: $72.5-85.1 \%)$ of 702 patients who underwent endoscopic surgery and $81.4 \%$ (95\% CI: $78.2-84.1 \%$ ) of 968 patients who underwent microsurgery $(P=0.583)$.

\section{http://www.endocrineconnections.org https://doi.org/10.1530/EC-17-0312}

2018 The authors Published by Bioscientifica Ltd

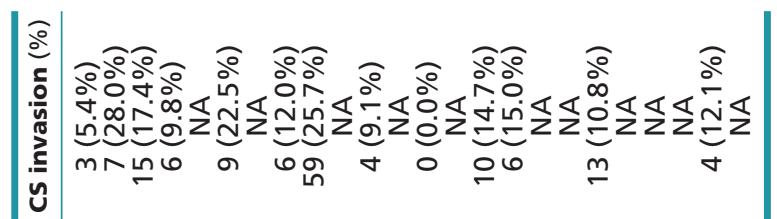

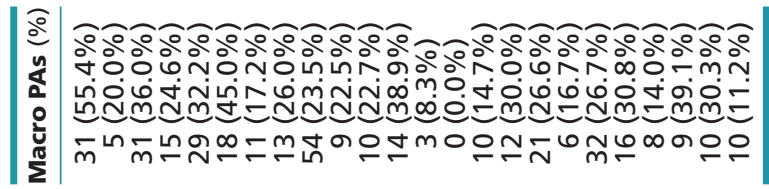

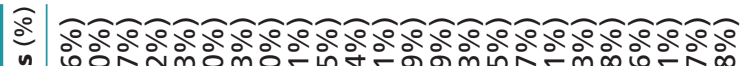

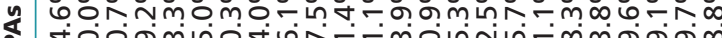

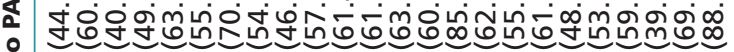

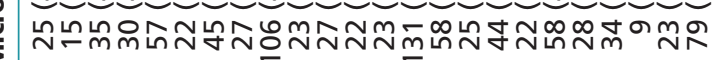

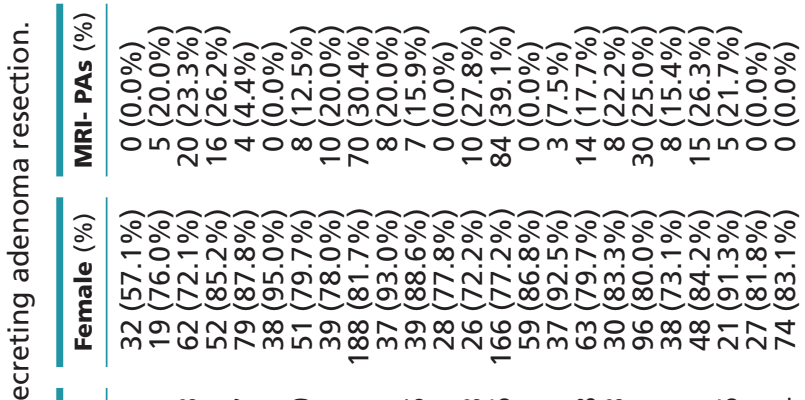

站

$\frac{7}{0}$

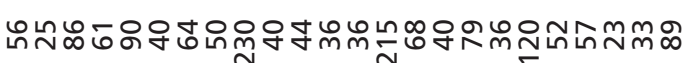

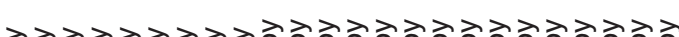

응증증증증증증증응응응응응응응응응응응응응응응

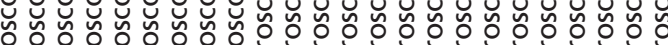

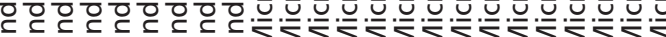

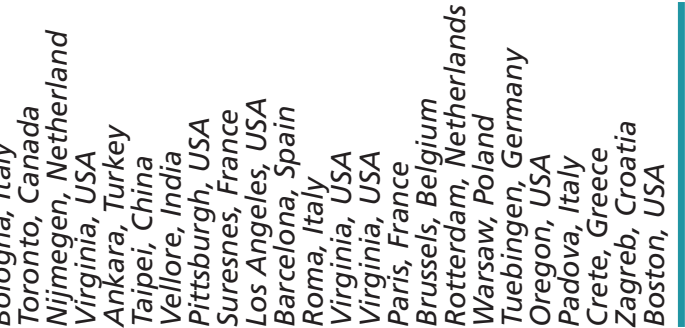
๓

\section{6}

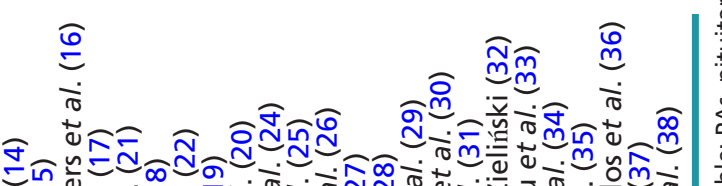

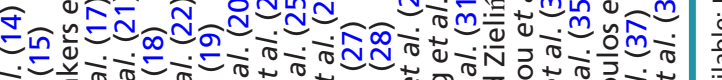

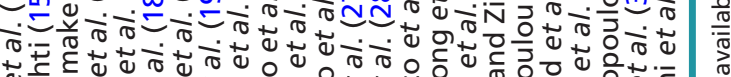

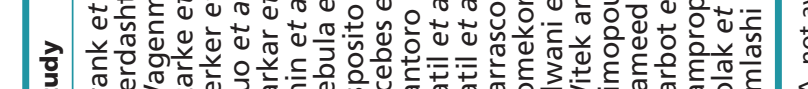

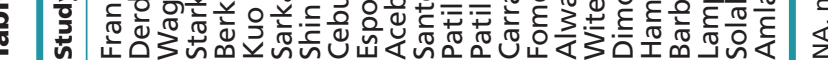

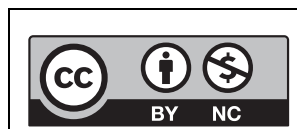

This work is licensed under a Creative Commons Attribution-NonCommercial 4.0 International License. 
More patients in the microscopic group (62.8\%) had micro-adenomas than did patients in the endoscopic group (53.1\%, 95\% CI: 56.1-69.1\% and 46.4-59.7\% respectively, $P=0.043)$. Conversely, more patients in the endoscopic group $(30.6 \%)$ had macroadenomas than did patients in the microscopic group $(22.0 \%, 95 \% \mathrm{CI}: 23.5-38.7 \%$ and $17.0-27.9 \%$ respectively, $P=0.066$ ). The proportion of MRI-negative tumors was nearly the same in both groups with $16.2 \%$ and $17.6 \%$ (95\% CI: $10.3-24.4 \%$ and $12.3-24.5 \%, P=0.769)$, respectively. We determined that $17.0 \%$ of patients treated endoscopically had cavernous sinus invasion compared with $11.9 \%$ of patients treated microsurgically (95\% CI: $11.6-24.2 \%$ and $8.8-16.0 \%$, $P=0.149$ ), though most of the studies did not supply these data.

\section{Outcome assessment}

There was no significant difference in remission proportion between the two groups, as shown in Fig. 1: $79.7 \% \quad(95 \%$ CI: $73.1-85.0 \%)$ of the patients who underwent endoscopic TS were in remission compared to $76.9 \%(95 \%$ CI: $71.3-81.6 \%)$ in the microscopic TS group ( $P=0.485$ ) (Table 2 ). There was no difference in the remission of magnetic resonance image (MRI)-negative tumors or of macroadenomas (Figs 2 and $3, P=0.229$ and $P=0.809$, respectively); however, the proportion of remission in micro-adenomas was significantly higher in the endoscopic group (87.3\%, 95\% CI: 83.2-90.5\%) than in the microscopic group (79.3\%, 95\% CI: 75.1-82.9\%, $P=0.004$, Fig. 4).

It seems that fewer patients who underwent endoscopic surgery recurred than did patients who underwent microscopic surgery (Fig. 5), with recurrence proportions of $11.0 \%$ and $15.9 \%$ (95\% CI: $7.6-15.7 \%$ and $11.5-21.7 \%$, respectively; $P=0.134$ ), respectively. However, if follow-up time is taken into account (36 months in endoscopic group and 53 months in microscopic group, $P=0.057$ ), both groups had a recurrence rate of approximately $4 \%$ per person per year (Fig. 6, 95\% CI: 3.1-5.4\% and $3.6-5.1 \%$, respectively; $P=0.651)$.

\section{Study}

Events Total

Method $=$ Endoscopy

G Frank (2006)

A Derdashti (2007)

M Wagenmakers (2013)

R Starke (2013)

M Berker (2014)

CH KUO (2015)

S Sarkar (2016)

S Shin (2017)

H Cebula (2017)

Random effects model

Heterogeneity: $I^{2}=68 \%, \tau^{2}=0.1974, p<0.01$

Method $=$ Microscopy

F Esposito (2006)

J Acebes (2007)

A Santoro (2007)

C Patil (2008)

C Patil (2008)

C Carrasco (2008)

E. Fomekong (2009)

A Alwani (2010)

P Witek (2012)

C Dimopoulou (2013)

N Hameed (2013)

M Barbot (2013)

K Lampropoulos (2013)

M Solak (2015)

F Amlashi (2015)

Random effects model

Heterogeneity: $I^{2}=68 \%, \tau^{2}=0.2033, p<0.01$

Random effects model

Heterogeneity: $I^{2}=67 \%, \tau^{2}=0.1905, p<0.01$

Test for subgroup differences: $\chi_{1}^{2}=0.49, \mathrm{df}=1(p=0.49)$
$38 \quad 56$

$62 \quad 86$

$58 \quad 61$

$81 \quad 90$

$47 \quad 64$

$39 \quad 50$

$192 \quad 230$

702

\section{$31 \quad 40$}

$39 \quad 44$

$27 \quad 36$

$22 \quad 36$

$\begin{array}{rr}184 & 215 \\ 50 & 68\end{array}$

$32 \quad 40$

$\begin{array}{ll}51 & 79\end{array}$

$23 \quad 36$

$\begin{array}{ll}85 & 120\end{array}$

$43 \quad 52$

$39 \quad 57$

$23 \quad 23$

$26 \quad 33$

\begin{tabular}{l}
$79 \quad 89$ \\
\hline
\end{tabular} 968

\section{0}

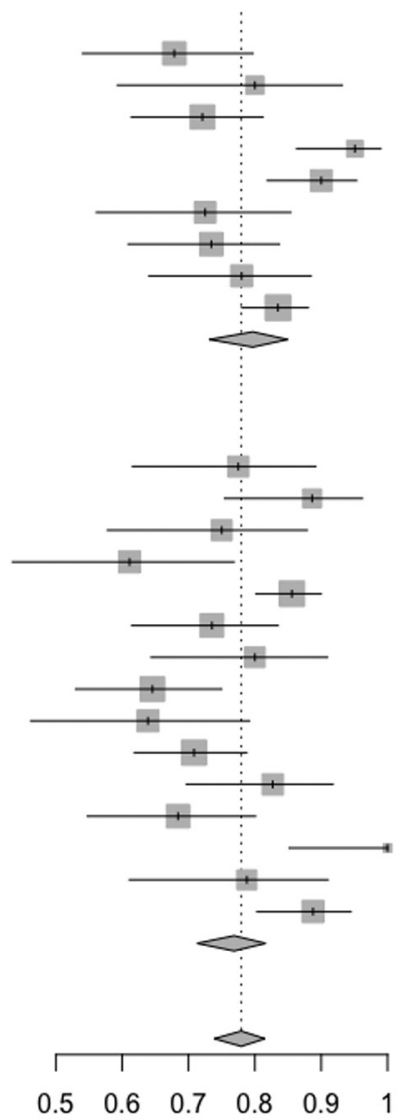

Proportion $\quad 95 \%-\mathrm{Cl}$ Weight

$\begin{array}{ll}0.68[0.54 ; 0.80] & 4.8 \% \\ 0.80[0.59 ; 0.93] & 2.9 \% \\ 0.72[0.61 ; 0.81] & 5.2 \% \\ 0.95[0.86 ; 0.99] & 2.4 \% \\ 0.90[0.82 ; 0.95] & 4.1 \% \\ 0.72[0.56 ; 0.85] & 4.1 \% \\ 0.73[0.61 ; 0.84] & 4.8 \% \\ 0.78[0.64 ; 0.88] & 4.2 \% \\ 0.83[0.78 ; 0.88] & 5.8 \% \\ 0.80[0.73 ; 0.85] & 38.4 \%\end{array}$

$0.78[0.62 ; 0.89] \quad 3.9 \%$

$0.89[0.75 ; 0.96] \quad 3.1 \%$

$0.75[0.58 ; 0.88] \quad 3.8 \%$

$0.61[0.43 ; 0.77] \quad 4.2 \%$

$0.86[0.80 ; 0.90] \quad 5.7 \%$

$0.74[0.61 ; 0.83] \quad 4.9 \%$

$0.80[0.64 ; 0.91] \quad 3.7 \%$

$0.65[0.53 ; 0.75] \quad 5.3 \%$

$0.64[0.46 ; 0.79] \quad 4.2 \%$

$0.71[0.62 ; 0.79] \quad 5.6 \%$

$0.83[0.70 ; 0.92] \quad 4.0 \%$

$0.68[0.55 ; 0.80] \quad 4.8 \%$

$1.00[0.85 ; 1.00] \quad 0.6 \%$

$0.79[0.61 ; 0.91] \quad 3.5 \%$

$0.89[0.80 ; 0.94] \quad 4.3 \%$

$0.77[0.71 ; 0.82] \quad 61.6 \%$

$0.78[0.74 ; 0.82] 100.0 \%$

Figure 1

Forest plot of remission proportion in the two groups.

http://www.endocrineconnections.org
https://doi.org/10.1530/EC-17-0312 Published by Bioscientifica Ltd

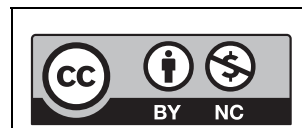

This work is licensed under a Creative Commons Attribution-NonCommercial 4.0 International License. 


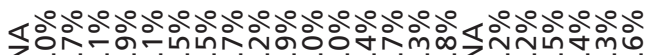

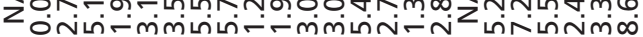

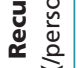
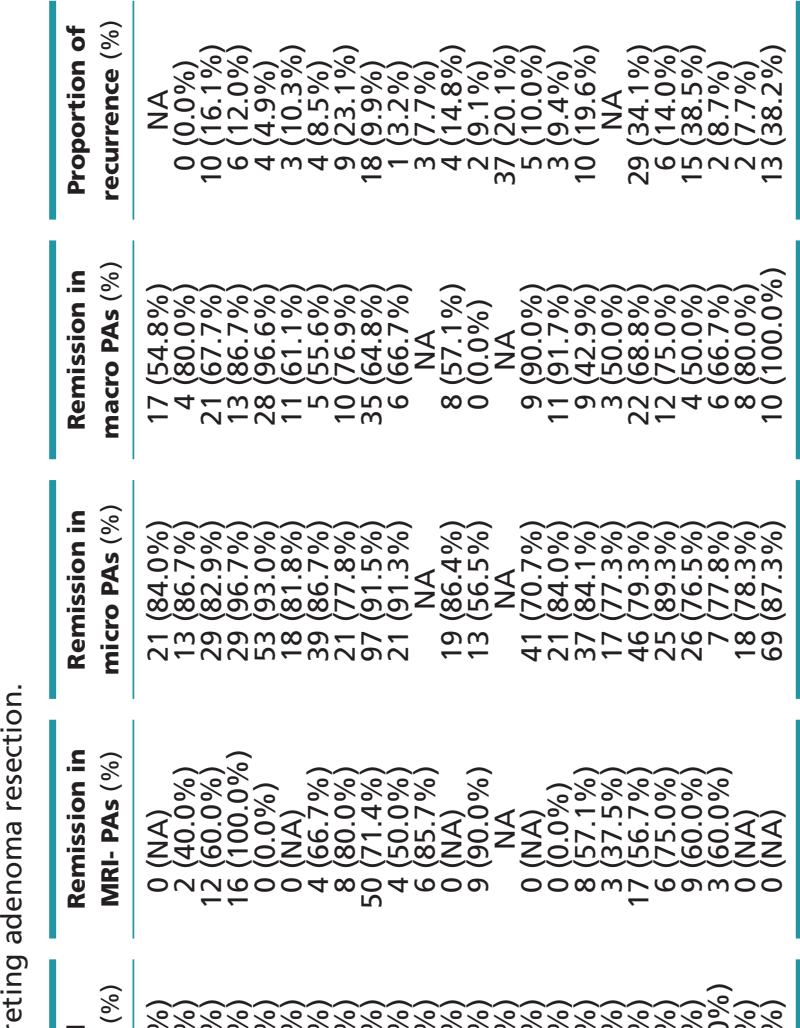

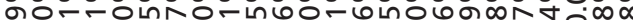
रेंนnं

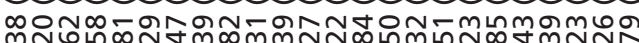

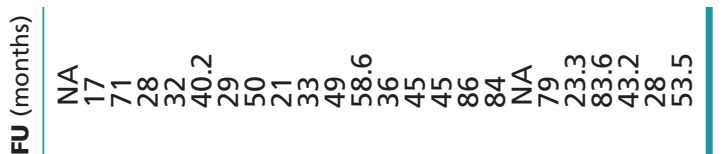

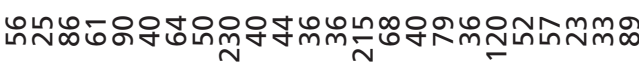

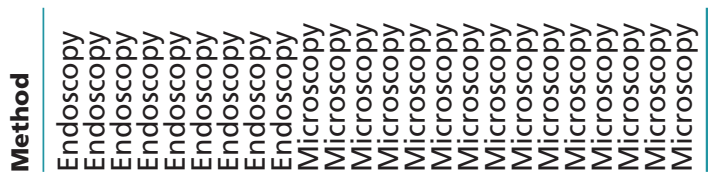

$\stackrel{\text { กิ }}{=}$

$\stackrel{=}{=}$

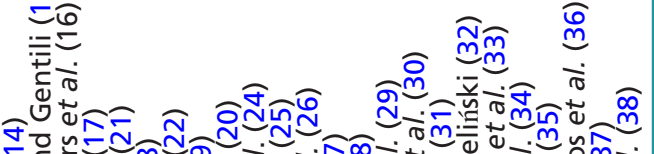


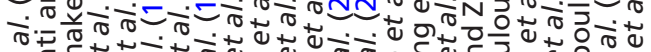

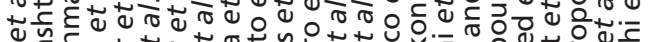

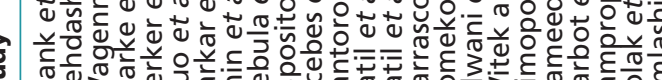

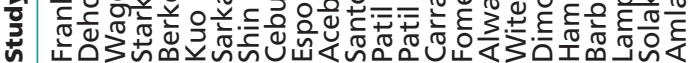

http://www.endocrineconnections.org https://doi.org/10.1530/EC-17-0312

2018 The authors Published by Bioscientifica Ltd
To investigate the effect of follow-up time on recurrence, we also included studies that reported patients treated prior to 1990 and with long-term follow-up (98 months compared to 53 months, respectively; $P=0.010$ ) (Table 3 ). There was no significant difference in remission between patients with long-term follow-up vs patients with relatively short follow-up times: $75.4 \%$ (95\% CI: $73.1-85.0 \%$ ) vs $76.9 \%$ (95\% CI: $71.3-81.6 \%, P=0.849$ ), respectively. Recurrence rate was also comparable between these two groups with $2.7 \%$ (95\% CI: $2.0-3.8 \%$ ) and $4.0 \%$ (95\% CI: $3.2-5.0 \%$ ) per person per year, respectively.

\section{Quality analysis}

Heterogeneity across studies was observed in the proportions of remission $\left(I^{2}=67 \%, \quad P<0.01\right)$ and recurrence $\left(I^{2}=67 \%, P<0.01\right)$. There was no indication of heterogeneity in the recurrence rate $\left(I^{2}=17 \%, P=0.23\right)$. To investigate the source of the heterogeneity, we conducted meta-regressions with several potential modifiers: number of patients, publication year, location in which the study was conducted and remission criteria. Our meta-regression analysis revealed no significant effects on the proportion of remission for publication date $(P=0.362)$, study location $(P=0.142)$, number of enrolled cases $(P=0.142)$ or remission criteria $(0.844)$. Publication date $(P=0.567)$, study location $(P=0.135)$ and number of enrolled cases $(P=0.440)$ did not contribute to the heterogeneity of recurrence proportion.

In the sensitivity analysis, a single study was removed to determine the influence of that individual data set on the pooled proportions or rates; the corresponding proportions and rates were not significantly altered, indicating that our results are statistically robust. Funnel plots were constructed to estimate the publication bias of the literature; the results suggest that any potential publication bias did not substantially influence the results of this meta-analysis.

\section{Discussion}

This systematic review and meta-analysis compares outcomes in endoscopic and microsurgical approaches for the treatment of ACTH-secreting pituitary adenomas. In our study, we found that basic characteristics of patients treated endoscopically were comparable to those of patients treated microscopically, except that more patients treated endoscopically had macroadenomas. Similar remission proportions were found for both endoscopic

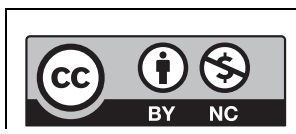

This work is licensed under a Creative Commons Attribution-NonCommercial 4.0 International License. 
Events Total

Proportion $\quad 95 \%-\mathrm{Cl}$ Weight

Method = Endoscopy

A Derdashti (2007)

M Wagenmakers (2013)

\section{5}

R Starke (2013)

M Berker (2014)

S Sarkar (2016)

S Shin (2017)

H Cebula (2017)

Fixed effect model

$\begin{array}{rr}12 & 20 \\ 16 & 16 \\ 0 & 4\end{array}$

04

$\begin{array}{rr}4 & 8 \\ 8 & 10\end{array}$

$50 \quad 70$

Heterogeneity: $I^{2}=49 \%, \tau^{2}=0.382, p=0.07$

Method $=$ Microscopy

F Esposito (2006)

J Acebes (2007)

C Patil (2008)

E. Fomekong (2009)

A Alwani (2010)

P Witek (2012)

C Dimopoulou (2013)

$\mathrm{N}$ Hameed (2013)

M Barbot (2013)

K Lampropoulos (2013)

Fixed effect model

Heterogeneity: $L^{2}=7 \%, \tau^{2}=0.037, p=0.37$

Fixed effect model

241

Heterogeneity: $I^{2}=30 \%, \tau^{2}=0.1704, p=0.11$

Subgoup test: $\chi_{1}^{2}=1.45, \mathrm{df}=1(p=0.23)$

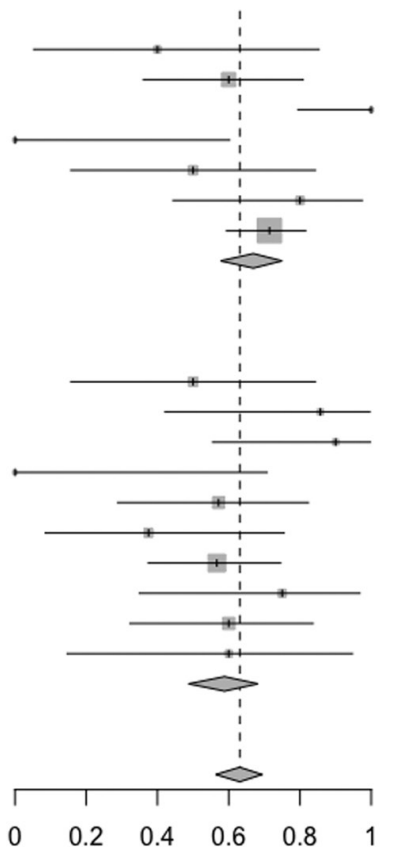

$0.40[0.05 ; 0.85] \quad 2.5 \%$

$0.60[0.36 ; 0.81] \quad 10.0 \%$

$1.00[0.79 ; 1.00] \quad 1.0 \%$

$0.00[0.00 ; 0.60] \quad 0.9 \%$

$0.50[0.16 ; 0.84] \quad 4.2 \%$

$0.80[0.44 ; 0.97] \quad 3.3 \%$

$0.71[0.59 ; 0.82] \quad 29.8 \%$

$0.67[0.58 ; 0.75] \quad 51.7 \%$

$0.50[0.16 ; 0.84] \quad 4.2 \%$

$0.86[0.42 ; 1.00] \quad 1.8 \%$

$0.90[0.55 ; 1.00] \quad 1.9 \%$

$0.00[0.00 ; 0.71] \quad 0.9 \%$

$0.57[0.29 ; 0.82] \quad 7.1 \%$

$0.38[0.09 ; 0.76] \quad 3.9 \%$

$0.57[0.37 ; 0.75] \quad 15.4 \%$

$0.75[0.35 ; 0.97] \quad 3.1 \%$

$0.60[0.32 ; 0.84] \quad 7.5 \%$

$0.60[0.15 ; 0.95] \quad 2.5 \%$

$0.59[0.49 ; 0.68] \quad 48.3 \%$

$0.63[0.56 ; 0.69] 100.0 \%$

Figure 2

Forest plot of remission proportion of MRI-negative adenomas in the two groups.

Study

Events Total

Method $=$ Endoscopy

$\begin{array}{lrr}\text { G Frank (2006) } & 17 & 31 \\ \text { A Derdashti (2007) } & 4 & 5 \\ \text { M Wagenmakers (2013) } & 21 & 31 \\ \text { R Starke (2013) } & 13 & 15 \\ \text { M Berker (2014) } & 28 & 29 \\ \text { CH KUO (2015) } & 11 & 18 \\ \text { S Sarkar (2016) } & 5 & 11 \\ \text { S Shin (2017) } & 10 & 13 \\ \text { H Cebula (2017) } & 35 & 54 \\ \text { Fixed effect model } & & 207\end{array}$

Heterogeneity: $I^{2}=44 \%, \tau^{2}=0.1989, p=0.07$

Method $=$ Microscopy

F Esposito (2006)

A Santoro (2007)

C Patil (2008)

C Carrasco (2008)

E. Fomekong (2009)

A Alwani (2010)

$P$ Witek (2012)

C Dimopoulou (2013)

$\mathrm{N}$ Hameed (2013)

M Barbot (2013)

K Lampropoulos (2013)

M Solak (2015)

F Amlashi (2015)

Fixed effect model

Heterogeneity: $I^{2}=36 \%, \tau^{2}=0.249, p=0.10$

Fixed effect model

367

Heterogeneity: $I^{2}=36 \%, \tau^{2}=0.1884, p=0.05$

Subgoup test: $\chi_{1}^{2}=0.06, \mathrm{df}=1(p=0.81)$

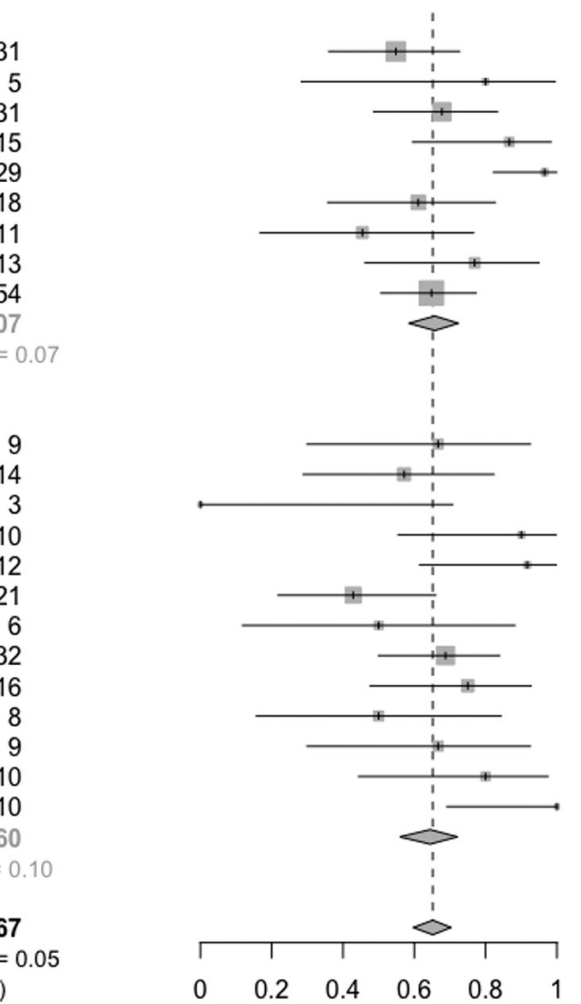

Proportion $\quad 95 \%-\mathrm{Cl}$ Weight

$\begin{array}{rr}0.55[0.36 ; 0.73] & 11.0 \% \\ 0.80[0.28 ; 0.99] & 1.1 \% \\ 0.68[0.49 ; 0.83] & 9.7 \% \\ 0.87[0.60 ; 0.98] & 2.5 \% \\ 0.97[0.82 ; 1.00] & 1.4 \% \\ 0.61[0.36 ; 0.83] & 6.1 \% \\ 0.45[0.17 ; 0.77] & 3.9 \% \\ 0.77[0.46 ; 0.95] & 3.3 \% \\ 0.65[0.51 ; 0.77] & 17.6 \% \\ 0.66[0.58 ; 0.72] & 56.7 \%\end{array}$

$0.67[0.30 ; 0.93] \quad 2.9 \%$

$0.57[0.29 ; 0.82] \quad 4.9 \%$

$0.00[0.00 ; 0.71] \quad 0.6 \%$

$0.90[0.55 ; 1.00] \quad 1.3 \%$

$0.92[0.62 ; 1.00] \quad 1.3 \%$

$0.43[0.22 ; 0.66] \quad 7.4 \%$

$0.50[0.12 ; 0.88] \quad 2.1 \%$

$0.69[0.50 ; 0.84] \quad 9.8 \%$

$0.75[0.48 ; 0.93] \quad 4.3 \%$

$0.50[0.16 ; 0.84] \quad 2.9 \%$

$0.67[0.30 ; 0.93] \quad 2.9 \%$

$0.80[0.44 ; 0.97] \quad 2.3 \%$

$1.00[0.69 ; 1.00] \quad 0.7 \%$

$0.64[0.56 ; 0.72] \quad 43.3 \%$

$0.65[0.60 ; 0.70] 100.0 \%$

Figure 3

Forest plot of remission proportion of macroadenomas in the two groups.

http://www.endocrineconnections.org

https://doi.org/10.1530/EC-17-0312
C) 2018 The authors Published by Bioscientifica Ltd

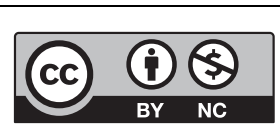

This work is licensed under a Creative Commons Attribution-NonCommercial 4.0 International License. 
Study

Events Total

Method = Endoscopy

G Frank (2006)

A Derdashti (2007)

M Wagenmakers (2013)

R Starke (2013)

M Berker (2014)

CH KUO (2015)

S Sarkar (2016)

S Shin (2017)

H Cebula (2017)

Fixed effect model

Heterogeneity: $I^{2}=10 \%$

Method = Microscopy

F Esposito (2006)

A Santoro (2007)

C Patil (2008)

C Carrasco (2008)

E. Fomekong (2009)

A Alwani (2010)

P Witek (2012)

C Dimopoulou (2013)

N Hameed (2013)

M Barbot (2013)

K Lampropoulos (2013)

M Solak (2015)

F Amlashi (2015)

Fixed effect model

Heterogeneity: $I^{2}=32 \%, \tau^{2}=0.091, p=0.13$

Fixed effect model

Heterogeneity: $I^{2}=39 \%, \tau^{2}=0.1383, p=0.03$

Subgoup test: $\chi_{1}^{2}=8.10, \mathrm{df}=1(p<0.01)$
810

25
15
35
30
57
22
45
27
06
62
$=0.35$

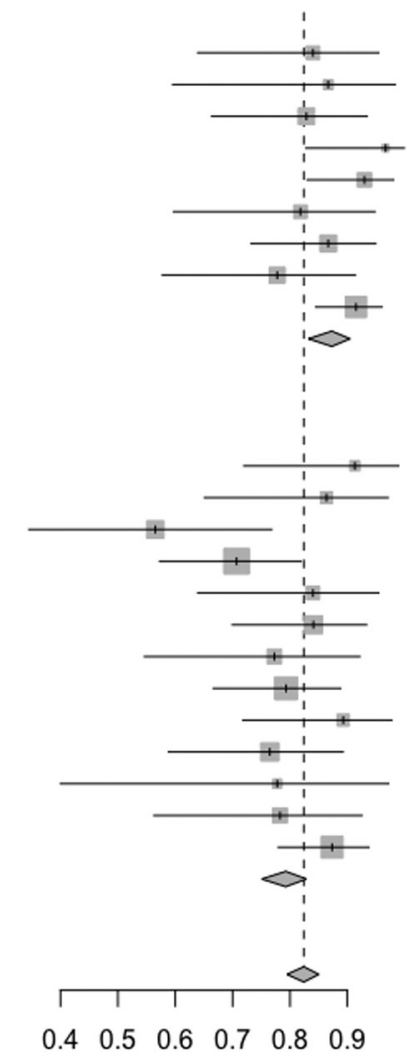

\section{Proportion $\quad 95 \%-\mathrm{Cl}$ Weight}

\begin{tabular}{|c|c|c|}
\hline & & \\
\hline & {$[0,0$} & \\
\hline & {$[0.66$} & \\
\hline & & \\
\hline & & \\
\hline 8 & {$[0.60 ; 0$} & \\
\hline & {$[0.7$} & \\
\hline & & \\
\hline & & \\
\hline & $.00,0$ & \\
\hline
\end{tabular}

$0.91[0.72 ; 0.99] \quad 1.8 \%$

$0.86[0.65 ; 0.97] \quad 2.5 \%$

$0.57[0.34 ; 0.77] \quad 5.4 \%$

$0.71[0.57 ; 0.82] 11.6 \%$

$0.84[0.64 ; 0.95] \quad 3.2 \%$

$0.84[0.70 ; 0.93] \quad 5.7 \%$

$0.77[0.55 ; 0.92] \quad 3.7 \%$

$0.79[0.67 ; 0.89] \quad 9.2 \%$

$0.89[0.72 ; 0.98] \quad 2.6 \%$

$0.76[0.59 ; 0.89] \quad 5.9 \%$

$0.78[0.40 ; 0.97] \quad 1.5 \%$

$0.78[0.56 ; 0.93] \quad 3.8 \%$

$0.87[0.78 ; 0.94] \quad 8.4 \%$

$0.79[0.75 ; 0.83] \quad 65.2 \%$

$0.82[0.79 ; 0.85] 100.0 \%$
Figure 4

Forest plot of remission proportion of microadenomas in the two groups.

and microsurgical approaches, though remission criteria differed from study to study. Patients treated with the endoscopic approach for micro-adenomas were more likely to achieve remission than those treated microsurgically. Recurrence seemed to be lower among patients treated endoscopically; however, when follow-up time is taken into account, this advantage disappears.

Because most of the studies with endoscopy were performed in the latest $10-15$ years. To eliminate the time as a confounding factor, we only included studies performed after 2005 (the oldest eligible publication on endoscopic TS is 2006). We only included studies with more than 20 patients because we believe surgical outcomes of CD are influenced by doctors' experience. On the other hand, we also performed sensitive analysis, even studies with less 20 patients were included, the result did not change. Studies with patients treated prior to 1990 were considered separately because the follow-up period was longer in these studies.

Endoscopic visualization provides a more panoramic view of the operative field, compared with the microscope, allowing for better viewing of the suprasellar region

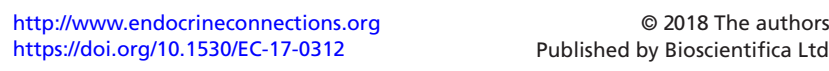

() 2018 The authors Published by Bioscientifica Ltd

$(51,52)$. It is also possible to use instruments with a variety of angles to access lateral invasions of tumors $(12,13)$. Endoscopic surgery is an excellent approach for patients with $\mathrm{CD}$, as the typically small size of the tumor requires higher magnification. Intrasellar illumination provided by the endoscope is extremely helpful in the intraoperative identification of abnormal tissue $(19,20,22,53)$. However, continuous adjustment of the endoscope is needed to determine target location within the surgical field, which may compromise maneuverability. Unlike endoscopic visualization, microsurgery offers a continuous view with a stereotactic display, which is familiar to the majority of surgeons and may allow for better control of bleeding in an open field. In recent years, some papers about 3D endoscopy for pituitary adenoma have been published $(67,68)$. This technique can combine depth perception in microscopy and wide-view in endoscopy. But no studies with $3 \mathrm{D}$ endoscopy were reported in patients with CD.

In the early days of endoscopic surgery, a large metaanalysis by Ammirati and coworkers (54) concluded that endoscopic removal of pituitary adenoma does not seem to confer any benefits over microscopic technology in

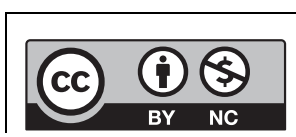

This work is licensed under a Creative Commons Attribution-NonCommercial 4.0 International License. 


\section{Events Total}

Method = Endoscopy

A Derdashti (2007)

M Wagenmakers (2013)

R Starke (2013)

M Berker (2014)

CH KUO (2015)

S Sarkar (2016)

S Shin (2017)

H Cebula (2017)

Random effects model

Heterogeneity: $I^{2}=42 \%, \tau^{2}=0.1319, p=0.10$

Method $=$ Microscopy

F Esposito (2006)

J Acebes (2007)

A Santoro (2007)

C Patil (2008)

C Patil (2008)

C Carrasco (2008)

E. Fomekong (2009)

A Alwani (2010)

C Dimopoulou (2013)

N Hameed (2013)

M Barbot (2013)

K Lampropoulos (2013)

M Solak (2015)

F Amlashi (2015)

Random effects model

Heterogeneity: $I^{2}=66 \%, \tau^{2}=0.2869, p<0.01$

Random effects model

1259

Heterogeneity: $I^{2}=67 \%, \tau^{2}=0.3211, p<0.01$

Test for subgroup differences: $\chi_{1}^{2}=2.25, \mathrm{df}=1(p=0.13)$
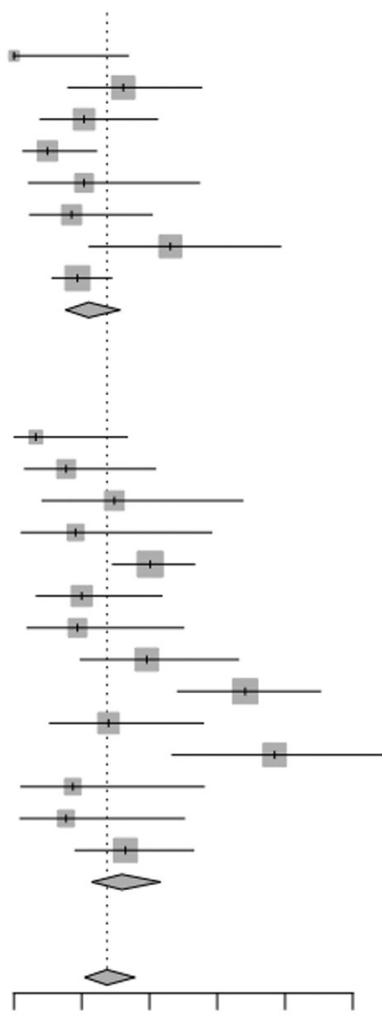

$\begin{array}{lllll}0.1 & 0.2 & 0.3 & 0.4 & 0.5\end{array}$

\section{Proportion 95\%-Cl Weight}

\begin{tabular}{|c|c|}
\hline$[0.00 ; 0.17]$ & \\
\hline $0.16[0.08 ; 0.28]$ & $5.8^{\circ}$ \\
\hline $0.10[0.04 ; 0.21]$ & 5.0 \\
\hline $.05[0.01 ; 0.12]$ & $4.4 \%$ \\
\hline $0.10[0.02 ; 0.27]$ & \\
\hline $09[0.02 ; 0.20]$ & \\
\hline$[0.11 ; 0.39]$ & \\
\hline$[0.06 ; 0.14]$ & \\
\hline$[0.08 ; 0.16]$ & \\
\hline
\end{tabular}

$0.03[0.00 ; 0.17] \quad 1.9 \%$

$0.08[0.02 ; 0.21] \quad 3.7 \%$

$0.15[0.04 ; 0.34] \quad 4.1 \%$

$0.09[0.01 ; 0.29] \quad 2.9 \%$

$0.20[0.15 ; 0.27] \quad 7.2 \%$

$0.10[0.03 ; 0.22] \quad 4.7 \%$

$0.09[0.02 ; 0.25] \quad 3.7 \%$

$0.20[0.10 ; 0.33] \quad 5.7 \%$

$0.34[0.24 ; 0.45] \quad 6.8 \%$

$0.14[0.05 ; 0.28] \quad 4.9 \%$

$0.38[0.23 ; 0.55] \quad 5.9 \%$

$0.09[0.01 ; 0.28] \quad 2.9 \%$

$0.08[0.01 ; 0.25] \quad 3.0 \%$

$0.16[0.09 ; 0.26] \quad 6.2 \%$

$0.16[0.11 ; 0.22] \quad 63.7 \%$

$0.14[0.10 ; 0.18] 100.0 \%$

\section{Figure 5}

Forest plot of recurrence proportion in the two groups.

the short term. However, recent meta-analyses showed that the endoscopic technique is associated with higher gross tumor removal (57) and modest increases of resection rates in residual or recurrent cases (58). In patients with functional pituitary adenomas (growth hormone-secreting adenoma), Phan and coworkers (59) concluded that clinical use of the endoscopic approach conferred potential benefits, including increased remission rates with non-invasive macroadenomas, but that overall endocrine remission is comparable. Chen and coworkers (60) also concluded that both approaches yielded similar rates of remission. However, a meta-analysis comparing outcomes from endoscopic TS and microscopic TS was lacking.

Our results support these findings. Overall remission, remission in macroadenomas, and remission in MRInegative CD showed no differences between groups. We also found that remission proportions for micro-adenomas were significantly higher in patients treated with the endoscopic approach compared to patients treated with the microsurgical approach. The superior intraoperative visualization afforded by the endoscopic approach may account for this finding. A much more unobstructed view of the operative field may facilitate resection of much of the tumor, especially the pseudocapsule $(61,62,63)$.

Complete surgical resection may be difficult for tumors with cavernous sinus infiltration due to the risks of injury of carotid artery and cranial nerves $(69,70)$. However, given the low occurrence of tumors with cavernous infiltration in each study, as well as the fact that few studies reported the remission of invasive tumors, the comparison between patients treated with endoscopic TS and microscopic TS was impossible in our analysis.

Regarding postoperative complications, previous studies include thorough descriptions and analyses, most of which demonstrate that patients who underwent endoscopic surgery had comparable proportions of complications, including diabetes insipidus, CSF leakage, hypocortisolemia, hypothyroidism, hypogonadism and visual defects, compared to patients who underwent microscopic surgery $(57,58,59,60)$. In this meta-analysis, we did not include any of these complications. Proportions http://www.endocrineconnections.org

https://doi.org/10.1530/EC-17-0312 (c) 2018 The authors Published by Bioscientifica Ltd

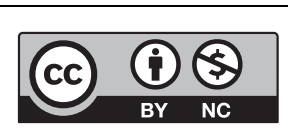

This work is licensed under a Creative Commons Attribution-NonCommercial 4.0 International License. 
Events Time

Method $=$ Endoscopy

$\begin{array}{lrr}\text { A Derdashti (2007) } & 0 & 28 \\ \text { M Wagenmakers (2013) } & 10 & 367 \\ \text { R Starke (2013) } & 6 & 135 \\ \text { M Berker (2014) } & 4 & 216 \\ \text { CH KUO (2015) } & 3 & 97 \\ \text { S Sarkar (2016) } & 4 & 114 \\ \text { S Shin (2017) } & 9 & 162 \\ \text { H Cebula (2017) } & 18 & 336\end{array}$

Fixed effect model

Heterogeneity: $I^{2}=0 \%, \tau^{2}=0.0003, p=0.43$

Method = Microscopy

F Esposito (2006) $\quad 1 \quad 85$

J Acebes (2007) $\quad 3159$

A Santoro (2007) $\quad 4 \quad 132$

C Patil (2008) $\quad 2 \quad 66$

C Patil (2008) $\quad 37 \quad 690$

C Carrasco (2008) $\quad 5 \quad 188$

E. Fomekong (2009) $\quad 3229$

A Alwani (2010) $\quad 10 \quad 357$

C Dimopoulou (2013) $\quad 29560$

N Hameed (2013) $\quad 6 \quad 83$

M Barbot (2013) $\quad 15 \quad 272$

K Lampropoulos (2013) 283

M Solak (2015) $\quad 2 \quad 61$

F Amlashi (2015) $\quad 13 \quad 352$

Fixed effect model

Heterogeneity: $I^{2}=28 \%, \tau^{2}=0.0465, p=0.15$

Fixed effect model

Heterogeneity: $I^{2}=17 \%, \tau^{2}=0.0259, p=0.23$

Subgoup test: $\chi_{1}^{2}=0.20, \mathrm{df}=1(p=0.65)$
Incidence Rate
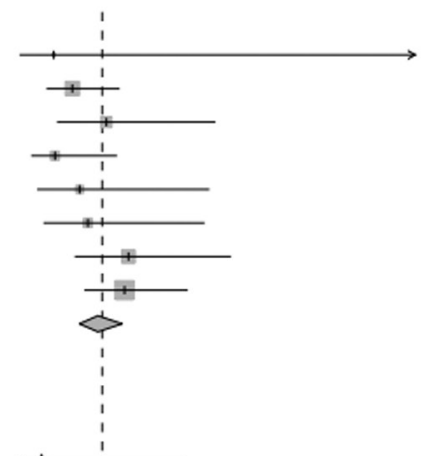

$0.01[0.00 ; 0.08] \quad 0.5 \%$

$0.02[0.01 ; 0.06] \quad 1.6 \%$

$0.03[0.01 ; 0.08] \quad 2.1 \%$

$0.03[0.01 ; 0.12] \quad 1.1 \%$

$0.05[0.04 ; 0.07] \quad 19.8 \%$

$0.03[0.01 ; 0.06] \quad 2.7 \%$

$0.01[0.00 ; 0.04] \quad 1.6 \%$

$0.03[0.02 ; 0.05] \quad 5.4 \%$

$0.05[0.04 ; 0.07] 15.5 \%$

$0.07[0.03 ; 0.16] \quad 3.2 \%$

$0.06[0.03 ; 0.09] \quad 8.0 \%$

$0.02[0.01 ; 0.10] \quad 1.1 \%$

$0.03[0.01 ; 0.13] \quad 1.1 \%$

$0.04[0.02 ; 0.06] \quad 7.0 \%$

$0.04[0.04 ; 0.05] \quad 70.8 \%$

$0.04[0.04 ; 0.05] 100.0 \%$

Figure 6

Forest plot of recurrence rate in the two groups.

of sinusitis and epistaxis were also comparable in previous reports $(59,64)$.

The definition of remission for CD varies over time and across studies. The remission of clinical symptoms, the need for glucocorticoid replacement, low or normal cortisol levels, normal 24-h urinary free cortisol levels, late-night salivary cortisol levels and cortisol after the dexamethasone suppression test all have been used in the literature $(14,15,16,17,18,19,20,21,22,24,25,26,27$, $28,29,30,31,32,33,34,35,36,37,38,39,40,41,42,43$, $44,45,46,47,48,49,50)$. A combination of two or three of the criteria mentioned earlier was used as the remission

Table 3 Studies with patients earlier than 1990 and with long-term follow-up.

\begin{tabular}{|c|c|c|c|c|c|c|c|}
\hline Study & Year & Place & Cases & $\begin{array}{l}\text { Follow-up } \\
\text { (months) }\end{array}$ & $\begin{array}{c}\text { Overall } \\
\text { remission (\%) }\end{array}$ & $\begin{array}{l}\text { Proportion of } \\
\text { recurrence (\%) }\end{array}$ & $\begin{array}{l}\text { Recurrence rate } \\
\text { (/person year) }\end{array}$ \\
\hline Valassi et al. (39) & 1982-2007 & Boston, USA & 620 & 47.4 & $477(76.9 \%)$ & $62(13.0 \%)$ & $3.3 \%$ \\
\hline Lindsay et al. (40) & 1982-2004 & Bethesda, USA & 418 & 125 & $331(79.2 \%)$ & $40(12.1 \%)$ & $1.2 \%$ \\
\hline Kim et al. (41) & 1984-2010 & Seoul, Korea & 54 & 104.6 & $38(70.4 \%)$ & $18(47.4 \%)$ & $5.4 \%$ \\
\hline Ciric et al. (42) & 1970-2010 & Chicago, Illinois & 136 & 68.4 & $93(68.4 \%)$ & $9(9.7 \%)$ & $1.7 \%$ \\
\hline Hassan-Smith et al. (43) & 1988-2009 & Birmingham, UK & 72 & 55.2 & $60(83.3 \%)$ & $8(13.3 \%)$ & $2.9 \%$ \\
\hline Lambert et al. (44) & 1980-2011 & New York, USA & 346 & 75.6 & $230(66.5 \%)$ & $73(31.7 \%)$ & $5.0 \%$ \\
\hline Alexandraki et al. (45) & 1969-2001 & London, UK & 131 & 180 & $86(65.6 \%)$ & $31(36.0 \%)$ & $2.4 \%$ \\
\hline Costenaro et al. (46) & 1989-2013 & Porto Alegre, Brazil & 103 & 73.2 & $84(81.6 \%)$ & $9(10.7 \%)$ & $1.8 \%$ \\
\hline Aranda et al. (47) & 1974-2011 & Barcelona, Spain & 41 & 168 & $32(78.0 \%)$ & $21(65.6 \%)$ & $4.7 \%$ \\
\hline Yamada et al. (48) & 1988-2014 & Tokyo, Japan & 230 & 72.5 & $198(86.1 \%)$ & $14(7.1 \%)$ & $1.2 \%$ \\
\hline Chandler et al. (49) & 1980-2012 & Michigan, USA & 275 & 80.4 & $219(79.6 \%)$ & $37(16.9 \%)$ & $2.5 \%$ \\
\hline Bansal et al. (50) & 1987-2015 & Maharashtra, India & 230 & 74 & $151(65.7 \%)$ & $48(31.8 \%)$ & $5.2 \%$ \\
\hline \multicolumn{2}{|c|}{$\begin{array}{l}\text { http://www.endocrineconnections.org } \\
\text { https://doi.org/10.1530/EC-17-0312 }\end{array}$} & $\begin{array}{r}\text { ๑ } 2018 \text { The authors } \\
\text { Published by Bioscientifica Ltd }\end{array}$ & & & \multicolumn{3}{|c|}{$\begin{array}{l}\text { This work is licensed under a Creative Commons } \\
\text { Attribution-NonCommercial } 4.0 \text { International } \\
\text { License. }\end{array}$} \\
\hline
\end{tabular}


criteria in our study. Due to improvements in biochemical assays, a new consensus holds more stringent criteria for remission (65): 'a postoperative cortisol value of $<2 \mathrm{mg} / \mathrm{dL}$ predicts a higher chance of long-term remission after TS in $\mathrm{CD}$; most patients with postoperative cortisol values of $2-5 \mathrm{mg} / \mathrm{dL}$ a few days after TS will also be in remission'. We also performed a subgroup analysis between studies with strict criteria and studies with lenient criteria. It turns out that no difference in remission proportion was observed in the two subgroups.

There was significant heterogeneity in the outcomes. This heterogeneity is likely impacted by differences in surgical technique, surgeon, team and institution experience or outcome criteria. It is also likely that differences in study design and definition of the outcomes influence heterogeneity (66).

A significant weakness of our analysis is that most studies use a relatively short follow-up time in patients with endoscopic TS. To compare recurrence rate between the two surgical groups, we assumed that there was no effect of follow-up time on recurrence rate. Metaregression showed that the slope of recurrence rate by follow-up time was minus $0.002(P=0.529)$, a trend suggesting that as follow-up time increases, recurrence rate may decrease. We found no studies that directly compared endoscopic and microsurgical approaches. Randomized trials with experienced surgeons and trials with long-term follow-up are required to help bridge the current gaps in the literature.

\section{Conclusion}

We found that overall remission proportion was the same in $\mathrm{CD}$ patients who underwent endoscopic TS compared to patients who underwent microscopic TS. However, patients treated with the endoscopic approach for micro-adenomas were more likely to achieve remission than those treated microsurgically. Patients treated endoscopically were less likely to experience recurrence; however, when follow-up time is taken into account, this advantage disappears. The definition of diagnosis, remission and recurrence is very challenging and variable, which has always to be considered in the interpretation of results of studies assessing therapeutic efficacy in CD.

\section{Declaration of interest}

The authors declare that there is no conflict of interest that could be perceived as prejudicing the impartiality of the research reported.

\section{Funding}

This study was supported by Shanghai Sailing Program (17YF1426700, 2017).

\section{Acknowledgements}

The authors thank Dr Min Shen and Dr Xuefei Shou for their contributions in the review of data.

\section{References}

1 Dallapiazza RF, Oldfield EH \& Jane JA. Surgical management of Cushing's disease. Pituitary 201518 211-216. (https://doi. org/10.1007/s11102-015-0646-5)

2 Tritos NA, Biller BMK \& Swearingen B. Management of Cushing disease. Nature Reviews Endocrinology 20117 279-289. (https://doi. org/10.1038/nrendo.2011.12)

3 Buchfelder M \& Schlaffer S. Pituitary surgery for Cushing's disease. Neuroendocrinology 201092 (Supplement 1) 102-106. (https://doi. org/10.1159/000314223)

4 Rutkowski MJ, Flanigan PM \& Aghi MK. Update on the management of recurrent Cushing's disease. Neurosurgical Focus 201538 E16. (https://doi.org/10.3171/2014.11.FOCUS14703)

5 Ayala A \& Manzano AJ. Detection of recurrent Cushing's disease: proposal for standardized patient monitoring following transsphenoidal surgery. Journal of Neuro-Oncology $2014119235-242$. (https://doi.org/10.1007/s11060-014-1508-0)

6 Pivonello R, De Martino MC, De Leo M, Simeoli C \& Colao A. Cushing's disease: the burden of illness. Endocrine 201756 10-18. (https://doi.org/10.1007/s12020-016-0984-8)

7 Fleseriu M. Medical management of persistent and recurrent Cushing disease. Neurosurgery Clinics of North America 201223 653-668. (https://doi.org/10.1016/j.nec.2012.06.012)

8 Yaneva M, Kalinov K \& Zacharieva S. Mortality in Cushing's syndrome: data from 386 patients from a single tertiary referral center. European Journal of Endocrinology 2013169 621-627. (https://doi.org/10.1530/EJE-13-0320)

9 van Haalen FM, Broersen LHA, Jorgensen JO, Pereira AM \& Dekkers OM. Management of endocrine disease: mortality remains increased in Cushing's disease despite biochemical remission: a systematic review and meta-analysis. European Journal of Endocrinology 2015172 R143-R149. (https://doi.org/10.1530/EJE-14-0556)

10 Clayton RN, Jones PW, Reulen RC, Stewart PM, Hassan-Smith ZK, Ntali G, Karavitaki N, Dekkers OM, Pereira AM, Bolland M, et al. Mortality in patients with Cushing's disease more than 10 years after remission: a multicentre, multinational, retrospective cohort study. Lancet Diabetes and Endocrinology 20164 569-576. (https://doi. org/10.1016/S2213-8587(16)30005-5)

11 Rolston JD, Han SJ \& Aghi MK. Nationwide shift from microscopic to endoscopic transsphenoidal pituitary surgery. Pituitary 201619 248-250. (https://doi.org/10.1007/s11102-015-0685-y)

12 Fernandez-Miranda JC, Zwagerman NT, Abhinav K, Lieber S, Wang EW, Snyderman CH \& Gardner PA. Cavernous sinus compartments from the endoscopic endonasal approach: anatomical considerations and surgical relevance to adenoma surgery. Journal of Neurosurgery 2017 [epub]. (https://doi.org/10.3171/2017.2. JNS162214)

13 Dusick JR, Esposito F, Kelly DF, Cohan P, DeSalles A, Becker DP \& Martin NA. The extended direct endonasal transsphenoidal approach for nonadenomatous suprasellar tumors. Journal of Neurosurgery 2005 102 832-841. (https://doi.org/10.3171/jns.2005.102.5.0832)

14 Frank G, Pasquini E, Farneti G, Mazzatenta D, Sciarretta V, Grasso V $\&$ Faustini Fustini M. The endoscopic versus the traditional approach

$$
\begin{array}{lr}
\text { http://www.endocrineconnections.org } & \odot 2018 \text { The authors } \\
\text { https://doi.org/10.1530/EC-17-0312 } & \text { Published by Bioscientifica Ltd }
\end{array}
$$

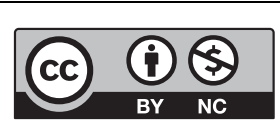


in pituitary surgery. Neuroendocrinology 200683 240-248. (https://doi.org/10.1159/000095534)

15 Dehdashti AR \& Gentili F. Current state of the art in the diagnosis and surgical treatment of Cushing disease: early experience with a purely endoscopic endonasal technique. Neurosurgical Focus 200723 E9-E8. (https://doi.org/10.3171/foc.2007.23.3.11)

16 Wagenmakers MAEM, Boogaarts HD, Roerink SHPP, Timmers HJLM, Stikkelbroeck NMML, Smit JWA, van Lindert EJ, Netea-Maier RT, Grotenhuis JA \& Hermus ARMM. Endoscopic transsphenoidal pituitary surgery: a good and safe primary treatment option for Cushing's disease, even in case of macroadenomas or invasive adenomas. European Journal of Endocrinology 2013169 329-337. (https://doi.org/10.1530/EJE-13-0325)

17 Starke RM, Reames DL, Chen C-J, Laws ER \& Jane JA Jr. Endoscopic transsphenoidal surgery for Cushing disease. Neurosurgery $2013 \mathbf{7 2}$ 240-247. (https://doi.org/10.1227/NEU.0b013e31827b966a)

18 Kuo C-H, Yen Y-S, Wu J-C, Chen Y-C, Huang W-C \& Cheng H. Primary endoscopic transnasal transsphenoidal surgery for magnetic resonance image-positive Cushing disease: outcomes of a series over 14 years. WNEU 201584 772-779. (https://doi.org/10.1016/j.wneu.2015.04.059)

19 Shin SS, Gardner PA, Ng J, Faraji AH, Agarwal N, Chivukula S, Fernandez-Miranda JC, Snyderman CH \& Challinor SM. Endoscopic endonasal approach for adrenocorticotropic hormone-secreting pituitary adenomas: outcomes and analysis of remission rates and tumor biochemical activity with respect to tumor invasiveness. WNEU 2017102 651.e1-658.e1. (https://doi.org/10.1016/j.wneu.2015.07.065)

20 Cebula H, Baussart B, Villa C, Assié G, Boulin A, Foubert L, Aldea S, Bennis S, Bernier M, Proust F, et al. Efficacy of endoscopic endonasal transsphenoidal surgery for Cushing's disease in 230 patients with positive and negative MRI. Acta Neurochirurgica 2017 1-10. (https://doi.org/10.1007/s00701-017-3140-1)

21 Berker M, Işikay I, Berker D, Bayraktar M \& Gürlek A. Early promising results for the endoscopic surgical treatment of Cushing's disease. Neurosurgical Review 201337 105-114. (https://doi.org/10.1007/ s10143-013-0506-6)

22 Sarkar S, Rajaratnam S, Chacko G, Mani S, Hesargatta AS \& Chacko AG. Pure endoscopic transsphenoidal surgery for functional pituitary adenomas: outcomes with Cushing's disease. Acta Neurochirurgica 2015 158 77-86. (https://doi.org/10.1007/s00701-015-2638-7)

23 Baker SG, Kramer BS \& Lindeman KS. The randomized registry trial. New England Journal of Medicine 2014370 681-682. (https://doi. org/10.1056/NEJMc1315677)

24 Esposito F, Dusick JR, Cohan P, Moftakhar P, McArthur D, Wang C, Swerdloff RS \& Kelly DF. Early morning cortisol levels as a predictor of remission after transsphenoidal surgery for Cushing's disease. Journal of Clinical Endocrinology and Metabolism 200691 7-13. (https://doi.org/10.1210/jc.2005-1204)

25 Acebes JJ, Martino J, Masuet C, Montanya E \& Soler J. Early postoperative ACTH and cortisol as predictors of remission in Cushing's disease. Acta Neurochirurgica 2007149 471-479. (https://doi. org/10.1007/s00701-007-1133-1)

26 Santoro A, Minniti G, Ruggeri A, Esposito V, Jaffrain-Rea M-L \& Delfini R. Biochemical remission and recurrence rate of secreting pituitary adenomas after transsphenoidal adenomectomy: long-term endocrinologic follow-up results. Surgical Neurology 200768 513-518. (https://doi.org/10.1016/j.surneu.2007.05.057)

27 Patil CG, Prevedello DM, Lad SP, Vance ML, Thorner MO, Katznelson L \& Laws ER Jr. Late recurrences of Cushing's disease after initial successful transsphenoidal surgery. Journal of Clinical Endocrinology and Metabolism 200893 358-362. (https://doi. org/10.1210/jc.2007-2013)

28 Patil CG, Veeravagu A, Prevedello DM, Katznelson L, Vance ML \& Laws ER Jr. Outcomes after repeat transsphenoidal surgery for recurrent Cushing's disease. Neurosurgery 200863 266-271. (https://doi.org/10.1227/01.NEU.0000313117.35824.9F)

29 Carrasco CA, Coste J, Guignat L, Groussin L, Dugué MA, Gaillard S, Bertagna X \& Bertherat J. Midnight salivary cortisol determination for assessing the outcome of transsphenoidal surgery in Cushing's disease. Journal of Clinical Endocrinology and Metabolism 200893 4728-4734. (https://doi.org/10.1210/jc.2008-1171)

30 Fomekong E, Maiter D, Grandin C \& Raftopoulos C. Outcome of transsphenoidal surgery for Cushing's disease: a high remission rate in ACTH-secreting macroadenomas. Clinical Neurology and Neurosurgery 2009111 442-449. (https://doi.org/10.1016/j.clineuro.2008.12.011)

31 Alwani RA, de Herder WW, van Aken MO, van den Berge JH, Delwel EJ, Dallenga AHG, de Jong FH, Lamberts SWJ, van der Lely AJ \& Feelders RA. Biochemical predictors of outcome of pituitary surgery for Cushing's disease. Neuroendocrinology 201091 169-178. (https://doi.org/10.1159/000258677)

32 Witek P \& Zieliński G. Predictive value of preoperative magnetic resonance imaging of the pituitary for surgical cure in Cushing's disease. Turkish Neurosurgery 2012 1-6. (https://doi.org/10.5137/10195149.JTN.6199-12.2)

33 Dimopoulou C, Schopohl J, Rachinger W, Buchfelder M, Honegger J, Reincke M \& Stalla GK. Long-term remission and recurrence rates after first and second transsphenoidal surgery for Cushing's disease: care reality in the Munich Metropolitan Region. European Journal of Endocrinology 2013170 283-292. (https://doi.org/10.1530/EJE-13-0634)

34 Hameed N, Yedinak CG, Brzana J, Gultekin SH, Coppa ND, Dogan A, Delashaw JB \& Fleseriu M. Remission rate after transsphenoidal surgery in patients with pathologically confirmed Cushing's disease, the role of cortisol, ACTH assessment and immediate reoperation: a large single center experience. Pituitary 201216 452-458. (https://doi.org/10.1007/s11102-012-0455-z)

35 Barbot M, Albiger N, Koutroumpi S, Ceccato F, Frigo AC, Manara R, Fassina A, Gardiman MP, Scanarini M, Mantero F, et al. Predicting late recurrence in surgically treated patients with Cushing's disease. Clinical Endocrinology 201379 394-401. (https://doi.org/10.1111/cen.12133)

36 Lampropoulos KI, Samonis G \& Nomikos P. Factors influencing the outcome of microsurgical transsphenoidal surgery for pituitary adenomas: a study on 184 patients. Hormones 201312 254-264. (https://doi.org/10.14310/horm.2002.1409)

37 Solak M, Kraljevic I, Dusek T, Melada A, Kavanagh MM, Peterkovic V, Ozretic D \& Kastelan D. Management of Cushing's disease: a single-center experience. Endocrine 201551 517-523. (https://doi. org/10.1007/s12020-015-0695-6)

38 Amlashi FG, Swearingen B, Faje AT, Nachtigall LB, Miller KK, Klibanski A, Biller BMK \& Tritos NA. Accuracy of late-night salivary cortisol in evaluating postoperative remission and recurrence in Cushing's disease. Journal of Clinical Endocrinology and Metabolism 2015100 3770-3777. (https://doi.org/10.1210/jc.2015-2107)

39 Valassi E, Biller BMK, Swearingen B, Pecori Giraldi F, Losa M, Mortini P, Hayden D, Cavagnini F \& Klibanski A. Delayed remission after transsphenoidal surgery in patients with Cushing's disease. Journal of Clinical Endocrinology and Metabolism 201095 601-610. (https://doi.org/10.1210/jc.2009-1672)

40 Lindsay JR, Oldfield EH, Stratakis CA \& Nieman LK. The postoperative basal cortisol and CRH tests for prediction of long-term remission from Cushing's disease after transsphenoidal surgery. Journal of Clinical Endocrinology and Metabolism 201196 2057-2064. (https://doi.org/10.1210/jc.2011-0456)

41 Kim JH, Shin CS, Paek SH, Jung H-W, Kim SW \& Kim SY. Recurrence of Cushing's disease after primary transsphenoidal surgery in a university hospital in Korea. Endocrine Journal 201259 881-888. (https://doi.org/10.1507/endocrj.EJ12-0109)

42 Ciric I, Zhao J-C, Du H, Findling JW, Molitch ME, Weiss RE, Refetoff S, Kerr WD \& Meyer J. Transsphenoidal surgery for Cushing disease. Neurosurgery 201270 70-81. (https://doi.org/10.1227/ NEU.0b013e31822dda2c)

43 Hassan-Smith ZK, Sherlock M, Reulen RC, Arlt W, Ayuk J, Toogood AA, Cooper MS, Johnson AP \& Stewart PM. Outcome of Cushing's disease following transsphenoidal surgery in a single center over 20 years. Journal of Clinical Endocrinology and Metabolism 201297 1194-1201. (https://doi.org/10.1210/jc.2011-2957) 
44 Lambert JK, Goldberg L, Fayngold S, Kostadinov J, Post KD \& Geer EB. Predictors of mortality and long-term outcomes in treated Cushing's disease: a study of 346 patients. Journal of Clinical Endocrinology and Metabolism 201398 1022-1030. (https://doi.org/10.1210/jc.2012-2893)

45 Alexandraki KI, Kaltsas GA, Isidori AM, Storr HL, Afshar F, Sabin I, Akker SA, Chew SL, Drake WM, Monson JP, et al. Long-term remission and recurrence rates in Cushing's disease: predictive factors in a single-centre study. European Journal of Endocrinology 2013168 639-648. (https://doi.org/10.1530/EJE-12-0921)

46 Costenaro F, Rodrigues TC, Rollin GAF, Ferreira NP \& Czepielewski MA. Evaluation of Cushing's disease remission after transsphenoidal surgery based on early serum cortisol dynamics. Clinical Endocrinology 201380 411-418. (https://doi.org/10.1111/cen.12300)

47 Aranda G, Enseñat J, Mora M, Puig-Domingo M, Martínez de Osaba MJ, Casals G, Verger E, Ribalta MT, Hanzu FA \& Halperin I. Long-term remission and recurrence rate in a cohort of Cushing's disease: the need for long-term follow-up. Pituitary 201418 142-149. (https://doi.org/10.1007/s11102-014-0567-8)

48 Yamada S, Inoshita N, Fukuhara N, Yamaguchi-Okada M, Nishioka H, Takeshita A, Suzuki H, Ito J \& Takeuchi Y. Therapeutic outcomes in patients undergoing surgery after diagnosis of Cushing's disease: a single-center study. Endocrine Journal 201562 1115-1125. (https://doi.org/10.1507/endocrj.15-0463)

49 Chandler WF, Barkan AL, Hollon T, Sakharova A, Sack J, Brahma B \& Schteingart DE. Outcome of transsphenoidal surgery for Cushing disease. Neurosurgery 201678 216-223. (https://doi.org/10.1227/ NEU.0000000000001011)

50 Bansal P, Lila A, Goroshi M, Jadhav S, Lomte N, Thakkar K, Goel A, Shah A, Sankhe S, Goel N, et al. Duration of post-operative hypocortisolism predicts sustained remission after pituitary surgery for Cushing's disease. Endocrine Connections 20176 625-636. (https://doi.org/10.1530/EC-17-0175)

51 Regmi D, Thapa A, Kc B \& Shakya B. Endoscopic endonasal approach. Journal of Nepal Health Research Council 201715 174-177. (https://doi.org/10.3126/jnhrc.v15i2.18209)

52 Song Y, Li H, Liu H, Li W, Zhang X, Guo L \& Tan G. Endoscopic endonasal transsphenoidal approach for sellar tumors beyond the sellar turcica. Acta Oto-Laryngologica 2014134 326-330. (https://doi org/10.3109/00016489.2013.857785)

53 Barkhoudarian G, Zada G \& Laws ER. Endoscopic endonasal surgery for nonadenomatous sellar/parasellar lesions. World Neurosurgery 201482 S138-S146. (https://doi.org/10.1016/j.wneu.2014.07.017)

54 Ammirati M, Wei L \& Ciric I. Short-term outcome of endoscopic versus microscopic pituitary adenoma surgery: a systematic review and meta-analysis. Journal of Neurology, Neurosurgery, and Psychiatry 201384 843-849. (https://doi.org/10.1136/jnnp-2012-303194)

55 Semple P. The transition from microscopic to endoscopic transsphenoidal surgery in high case load neurosurgical centers: the Groote Schuur Hospital experience. World Neurosurgery 201482 S162-S163. (https://doi.org/10.1016/j.wneu.2014.08.002)

56 Laws ER \& Barkhoudarian G. The transition from microscopic to endoscopic transsphenoidal surgery: the experience at Brigham and Women's Hospital. World Neurosurgery 201482 S152-S154. (https://doi.org/10.1016/j.wneu.2014.07.035)

57 Li A, Liu W, Cao P, Zheng Y, Bu Z \& Zhou T. Endoscopic versus microscopic transsphenoidal surgery in the treatment of pituitary adenoma: a systematic review and meta-analysis. World Neurosurgery 2017101 236-246. (https://doi.org/10.1016/j.wneu.2017.01.022)

58 Esquenazi Y, Essayed WI, Singh H, Mauer E, Ahmed M, Christos PJ \& Schwartz TH. Endoscopic endonasal versus microscopic transsphenoidal surgery for recurrent and/or residual pituitary transsphenoidal approach to pituitary adenoma: a multi-disciplinary

adenomas. World Neurosurgery 2017101 186-195. (https://doi. org/10.1016/j.wneu.2017.01.110)

59 Phan K, Xu J, Reddy R, Kalakoti P, Nanda A \& Fairhall J. Endoscopic endonasal versus microsurgical transsphenoidal approach for growth hormone-secreting pituitary adenomas-systematic review and meta-analysis. WNEU 201797 398-406. (https://doi.org/10.1016/j. wneu.2016.10.029)

60 Chen C-J, Ironside N, Pomeraniec IJ, Chivukula S, Buell TJ, Ding D, Taylor DG, Dallapiazza RF, Lee C-C \& Bergsneider M. Microsurgical versus endoscopic transsphenoidal resection for acromegaly: a systematic review of outcomes and complications. Acta Neurochirurgica 2017159 2193-2207. (https://doi.org/10.1007/ s00701-017-3318-6)

61 Monteith SJ, Starke RM, Jane JA \& Oldfield EH. Use of the histological pseudocapsule in surgery for Cushing disease: rapid postoperative cortisol decline predicting complete tumor resection. Journal of Neurosurgery 2012116 721-727. (https://doi. org/10.3171/2011.12.JNS11886)

62 Taylor DG, Jane JA \& Oldfield EH. Resection of pituitary macroadenomas via the pseudocapsule along the posterior tumor margin: a cohort study and technical note. Journal of Neurosurgery 2017 1-7. (https://doi.org/10.3171/2017.7.JNS171658)

63 Ceylan S, Cabuk B, Koc K, Anik I \& Vural C. Endoscopic distinction between capsule and pseudocapsule of pituitary adenomas. Acta Neurochirurgica 2013155 1611-1619. (https://doi.org/10.1007/ s00701-013-1754-5)

64 Lenzi J, Lapadula G, D'amico T, Delfinis CP, Iuorio R, Caporlingua F, Mecca N, Mercuri V, Bassotti G, Rillo M, et al. Evaluation of trans-sphenoidal surgery in pituitary GH-secreting micro- and macroadenomas: a comparison between microsurgical and endoscopic approach. Journal of Neurosurgical Sciences 201559 11-18.

65 Fleseriu M, Hamrahian AH, Hoffman AR, Kelly DF, Katznelson L, AACE Neuroendocrine \& Pituitary Scientific Committee*. American Association of Clinical Endocrinologists and American College of Endocrinology disease state clinical review: diagnosis of recurrence in Cushing disease. Endocrine Practice 201622 1436-1448. (https://doi. org/10.4158/EP161512.DSCR)

66 Petersenn S, Beckers A, Ferone D, van der Lely A, Bollerslev J, Boscaro M, Brue T, Bruzzi P, Casanueva FF, Chanson P, et al. Therapy of endocrine disease: outcomes in patients with Cushing's disease undergoing transsphenoidal surgery: systematic review assessing criteria used to define remission and recurrence. European Journal of Endocrinology 2015 172 R227-R239. (https://doi.org/10.1530/EJE-14-0883)

67 Pennacchietti V, Garzaro M, Grottoli S, Pacca P, Garbossa D, Ducati A \& Zenga F. Three-dimensional endoscopic endonasal approach and outcomes in sellar lesions: a single-center experience of 104 cases. World Neurosurgery 201689 121-125. (https://doi.org/10.1016/j. wneu.2016.01.049)

68 Rampinelli V, Doglietto F, Mattavelli D, Qiu J, Raffetti E, Schreiber A, Villaret AB, Kucharczyk W, Donato F, Fontanella MM, et al. TwoDimensional high definition versus three-dimensional endoscopy in endonasal skull base surgery: a comparative preclinical study. World Neurosurgery 2017105 223-231. (https://doi.org/10.1016/j. wneu.2017.05.130)

69 Smith TR, Hulou MM, Huang KT, Nery B, de Moura SM, Cote DJ \& Laws ER. Complications after transsphenoidal surgery for patients with Cushing's disease and silent corticotroph adenomas. Neurosurgical Focus 201538 E12. (https://doi.org/10.3171/2014.10.FOCUS14705)

70 Gardner PA, Tormenti MJ, Pant H, Fernandez-Miranda JC, Snyderman CH \& Horowitz MB. Carotid artery injury during endoscopic endonasal skull base surgery: incidence and outcomes. Neurosurgery 201373 261-269.

Received in final form 22 November 2017 Accepted 28 November 2017 Attribution-NonCommercial 4.0 International

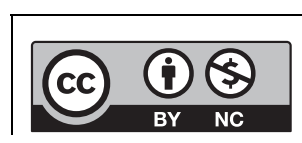

This work is licensed under a Creative Commons License. 